\title{
Perceived risk of harm and intentions of future inhalant use among adolescent inhalant users
}

\author{
Brian E. Perron ${ }^{\mathrm{a}, *}$, Matthew O. Howard ${ }^{\mathrm{b}}$ \\ ${ }^{a}$ University of Michigan, School of Social Work, 1080 South University Avenue, Ann Arbor, MI 48103, United States \\ ${ }^{\mathrm{b}}$ University of North Carolina at Chapel Hill, School of Social Work, Tate-Turner-Kuralt Building, 325 Pittsboro Street, \\ CB \#3550, Chapel Hill, NC 27599-3550, United States
}

Received 22 October 2007; received in revised form 11 April 2008; accepted 12 April 2008

Available online 3 June 2008

\begin{abstract}
Objective: To identify predictors of perceived (a) risk of harm associated with inhalant use and (b) intention to use inhalants among adolescent inhalant users.

Method: Participants were 279 lifetime inhalant users $\left(M_{\mathrm{age}}=15.5,84 \%\right.$ male) identified in a statewide survey of 723 adolescents in Missouri Division of Youth Services' residential care for antisocial conduct. Youth completed interviews assessing inhalant and other drug use, psychiatric symptoms, and antisocial traits/behavior.

Results: More than one-third (37\%) of youth perceived experimental inhalant use as of slight or no risk; one-in-eight (11.9\%) youth perceived regular inhalant use as of slight or no risk. Risk perceptions of experimental and regular inhalant use were not associated with intentions to use. Youth with friends/siblings who use inhalants were less likely to perceive risks associated with experimental and regular inhalant use compared to youth without friends/sibling users. Adolescents who were younger and those with more extensive substance abuse problems, prior problems with inhalants, greater current psychiatric distress, and friends/siblings who use inhalants were significantly more likely to report intentions of future inhalant use than their counterparts.

Conclusions: Assessments of substance use among youth, particularly those in the criminal justice system, should include an assessment of inhalant use. Intervention efforts should focus on developing strategies for managing social network influences.
\end{abstract}

(C) 2008 Elsevier Ireland Ltd. All rights reserved.

Keywords: Youth; Delinquency; Inhalants; Volatile solvents

\section{Introduction}

Inhalant use is among the most pernicious and least studied forms of adolescent drug abuse (Howard et al., 2008). Approximately 2 million U.S. adolescents use inhalants annually (Wu et al., 2004). In 2006, $16.1 \%$ of U.S. 8th graders reported prior inhalant use (Johnson et al., 2007). Despite widespread use among adolescents, inhalant abuse has been called the "forgotten drug problem" due to the limited research historically accorded to this class of agents (Balster, 1997; Perron et al., in press; Vaughn et al., 2007).

\footnotetext{
* Corresponding author.

E-mail addresses: beperron@umich.edu (B.E. Perron), mohoward@email.unc.edu (M.O. Howard).
}

Several studies have examined predictors of initiation of inhalant use (e.g., Howard et al., 1999), but few investigations have evaluated the extent to which adolescent inhalant users consider inhalant use a potentially risky activity and factors associated with inhalant-related risk perceptions. Further, the role that risk perceptions play in adolescent inhalant users' expectancies for future inhalant use is presently unclear, although limited findings suggest that perceptions of harm are inversely related to inhalant use (e.g., Beauvais, 1992). Recent research indicates that self-reported intentions to use inhalants are significantly related to future inhalant use, consistent with the theory of reasoned action (Crano et al., in press). Thus, studies identifying correlates of inhalant-related risk perceptions and intentions to use inhalants may provide valuable information to guide prevention and treatment programming in this increasingly important area. 
Juvenile offenders are an important population in which to study inhalant use. Inhalant use and its consequences are prevalent in many subpopulations of youth exhibiting delinquent behaviors and can be readily assessed when youth are in residential care and free of inhalant use and acute substance-related functional impairments (Howard and Jenson, 1999). In a recently completed survey of Missouri youth court ordered into residential treatment for antisocial behavior (which forms the basis for this report), $38.6 \%$ of 723 youth were identified as lifetime inhalant users (Howard et al., 2008). Besides being problematic in the United States, inhalant use is prevalent among children and adolescents in many other nations. Medina-Mora and Real (2008) reviewed recently reported epidemiological studies of inhalant use among citizens of Brazil, Mexico, Paraguay, Chile, Columbia, Nicaragua, Spain, Canada, New Zealand, and Australia, concluding that inhalant use is "widespread among children and adolescents and is increasing among females in the developing and developed world [and associated with] increased risk of injection drug use, HIV, suicidality, and psychiatric disorders among inhalant users" (p. 247). High rates of inhalant use and inhalant-related problems have also recently been identified among Russian street youth (Kissin et al., 2007), Japanese high school students (Shimane and Wada, 2007), and youth in eastern European countries. Greater knowledge as to the correlates of inhalant-related risk perceptions and intentions/expectancies to use inhalants could do much to improve the treatment of adolescent inhalant users, especially those treated in juvenile justice and other rehabilitative settings.

We hypothesized that youth with comparatively high levels of antisocial behavior and attitudes, substance abuse problems, self-reported prior problems with inhalants, friends or sibling who use inhalants, and otherwise high-risk sociodemographic profiles would report lower levels of perceived risk of harm in association with experimental and regular inhalant use. We further hypothesized that these factors, in conjunction with lower levels of perceived risk of harm in association with inhalant use, would be associated with an increased perceived likelihood of post-discharge inhalant use.

\section{Methods}

\subsection{Study sample}

Findings of the present study are based on a survey conducted in 2003 of the population of current residents of the Missouri Division of Youth Services' (MDYS) residential rehabilitation system. A detailed description of the study sample and survey is available in Howard et al. (2008). The MDYS is the legal guardian of all residents who are committed to its care by the state's 45 juvenile courts. The 723 adolescents who completed the interview constituted $97.7 \%$ of MDYS residents at the time interviewing was conducted and 55.0\% of youth committed to MDYS care in the prior year. The informed consent and study protocols were approved by the Missouri DYS and Washington University IRBs and Office of Human Research Protection.

\subsection{Measures}

All participants completed the Volatile Solvent Screening Inventory (VSSI), an interview assessing demographic characteristics, medical history, lifetime/annual use of 65 inhalants, other drug use and substance-related problems, current psychiatric symptoms, thoughts of suicide/actual suicide attempts, trauma history, antisocial traits and criminal activity. All youth reporting any lifetime use of an inhalant in an effort to get high also completed the Comprehensive Solvent Assessment Interview (CSAI), a schedule evaluating reasons for starting and stopping inhalant use, typical modes, locations, contexts and subjective effects of inhalant use, adverse consequences/high-risk behaviors occurring in association with acute inhalant intoxication, perceived risks of inhalant use, estimated likelihood that inhalants will be used in the future, sibling and friend's use of inhalants, and DSM-IV Inhalant Abuse and Dependence criteria. This study included all youth reporting lifetime use of inhalants to get high $(n=279)$.

2.2.1. Perceived risk of harm of inhalant use. Youth were asked how much they think people risk harming themselves physically and in other ways if they try inhalants once or twice (experimental use), and if they use inhalants regularly (regular use). Response options for the two perceived risk questions were: 0 , no risk; 1 , slight risk; 2 , medium risk; 3 , great risk.

2.2.2. Intentions of future inhalant use. Youth who had used one or more volatile solvent inhalants (i.e., all participants in this study) were asked: "During the next year (after you are released from custody) how likely are you to use inhalants?" Responses were recorded on a five-point Likert-type scale ( 0 : no chance, 1: little chance, 2: an even chance, 3: a good chance, 4: I am sure to).

2.2.3. Inhalant and other substance-related measures. Youth were asked whether they had ever "had a problem" with inhalants before they entered custody (0: no vs. 1: yes). Inhalant use within a youth's social network reflected whether any of the respondents' friends (yes/no) or siblings (yes/no) currently used inhalants. Lifetime substance-related problems were assessed with the 8-item Alcohol/Drug Use Scale of the Massachusetts Adolescents Screening Instrument-2nd Version (MAYSI-2) (Grisso and Barnum, 2000). Scores could range from 0 to 8 , with higher values representing a greater number of problems.

2.2.4. Psychiatric variables. Youth completed the Brief Symptom Inventory (BSI), consisting of 53 items assessing the extent to which they were "bothered or disturbed" ( 0 : not at all; 4: extremely) by a variety of thoughts or feelings "over the last 7 days including today" (Derogatis, 1993). A global severity index (GSI) score was computed by summing scores for all items. Antisocial attitudes were assessed using the total score of the Psychopathic Personality Inventory (PPI; Lilienfeld and Andrews, 1996) and the Self-Rerpot of Delinquency (Elliott et al., 1989).

2.2.5. Demographic and psychosocial variables. Gender, age, self-reported racial status, family receipt of public assistance, and geographical area of family residence (i.e., urban, suburban, small town, rural) were recorded for each youth. Youth were also asked to report the duration (months) of their current residential treatment episode.

\subsection{Analytic procedures}

Descriptive statistics were used to summarize results. Given the skewed distributions of the dependent measures, non-parametric correlations were computed (i.e., Spearman rank-order correlation). Ordinal logistic regression was used to test multivariate associations and study hypotheses. Adjusted odds ratios (OR) and 95\% confidence intervals were computed to indicate statistical significance and effect sizes.

\section{Results}

\subsection{Characteristics of inhalant user sample}

Approximately $82 \%(n=230)$ of the sample reported a "White" racial identification and $18 \%(n=49)$ was non-White. Eighty-four percent of youth were male, and ages ranged from 11 to $20($ mean $=15.5$, S.D. $=1.2)$. Thirty-nine percent $(n=109)$ of youth reported that their family currently received public assistance. Prior to being ordered into residential care, 59\% of respondents $(n=166)$ lived in small town/rural areas and $41 \%$ $(n=113)$ lived in urban/suburban areas. The current episode of 
Table 1

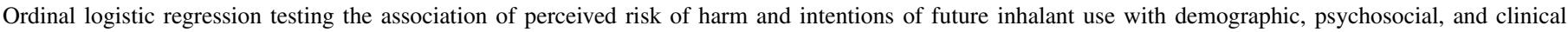
measures

\begin{tabular}{|c|c|c|c|c|}
\hline Variables & $\begin{array}{l}\text { Univariate summary } \\
\text { measures }\end{array}$ & $\begin{array}{l}\text { Perceived risk of harm with } \\
\text { experimental inhalant use }{ }^{a} \text {, } \\
\text { AOR }(95 \% \mathrm{CI})\end{array}$ & $\begin{array}{l}\text { Perceived risk of harm with } \\
\text { regular inhalant use }{ }^{b}, \text { AOR } \\
(95 \% \mathrm{CI})\end{array}$ & $\begin{array}{l}\text { Intentions of future inhalant } \\
\text { use }^{c} \text { AOR }(95 \% \mathrm{CI})\end{array}$ \\
\hline Age $_{11-20}$ (in years) & $15.5(1.2)$ & $.96(.78-1.19)$ & $1.00(.78-1.29)$ & $.74(.56-.99)$ \\
\hline \multicolumn{5}{|l|}{ Gender } \\
\hline Female & $16 \%, n=44$ & Ref & Ref & Ref \\
\hline Male & $84 \%, n=235$ & $1.23(.68-2.20)$ & $1.65(.77-3.56)$ & $.82(.37-1.81)$ \\
\hline \multicolumn{5}{|l|}{ Ethnicity } \\
\hline Non-White & $18 \%, n=49$ & Ref & Ref & Ref \\
\hline White & $82 \%, n=230$ & $.81(.47-1.40)$ & $.68(.34-1.35)$ & $1.00(.48-2.07)$ \\
\hline \multicolumn{5}{|l|}{ Public assistance } \\
\hline No & $61 \%, n=170$ & Ref & Ref & Ref \\
\hline Yes & $39 \%, n=109$ & $1.06(.67-1.66)$ & $1.24(.71-2.17)$ & $.64(.34-1.21)$ \\
\hline \multicolumn{5}{|l|}{ Urbanicity } \\
\hline Rural/small town & $59 \%, n=166$ & Ref & Ref & Ref \\
\hline Urban/suburban & $41 \%, n=113$ & $1.08(.68-1.72)$ & $1.23(.71-2.16)$ & $.69(.37-1.29)$ \\
\hline Months of incarceration & $7.5(8.5)$ & $1.09(.88-1.34)$ & $1.11(.84-1.46)$ & $.89(.65-1.22)$ \\
\hline \multicolumn{5}{|l|}{ Perceived problem with inhalants } \\
\hline No & $62 \%, n=173$ & Ref & Ref & Ref \\
\hline Yes & $38 \%, n=106$ & $1.13(.71-1.81)$ & $1.25(.71-2.20)$ & $2.39(1.27-4.49)$ \\
\hline MAYSI-2 - substance use problems index & $5.2(2.1)$ & $1.13(.69-1.85)$ & $1.31(.73-2.34)$ & $2.13(1.01-4.50)$ \\
\hline \multicolumn{5}{|l|}{ Perceived harm of inhalants - single use } \\
\hline No risk & $10 \%, n=29$ & $\dagger$ & $\dagger$ & Ref \\
\hline Slight risk & $27 \%, n=76$ & & & $.57(.15-2.29)$ \\
\hline Medium risk & $24 \%, n=67$ & & & $1.71(.38-7.66)$ \\
\hline Great risk & $38 \%, n=107$ & & & $.23(.05-1.07)$ \\
\hline \multicolumn{5}{|l|}{ Perceived harm of inhalants - regular use } \\
\hline No risk & $4.7 \%, n=13$ & $\dagger$ & $\dagger$ & Ref \\
\hline Slight risk & $7.2 \%, n=20$ & & & $.50(.07-3.85)$ \\
\hline Medium risk & $18.0 \%, n=49$ & & & $.61(.09-3.90)$ \\
\hline Great risk & $71.0 \%, n=197$ & & & $.99(.15-6.63)$ \\
\hline \multicolumn{5}{|l|}{ Friends or siblings use inhalants } \\
\hline No & $63 \%, n=186$ & Ref & Ref & Ref \\
\hline Yes & $37 \%, n=93$ & $.55(.34-.89)$ & $.47(.26-.82)$ & 3.33 (1.77-6.29) \\
\hline Global Severity Index ${ }^{\ddagger}$ & $53.82(37.16)$ & $1.40(.96-2.04)$ & $1.35(.87-2.10)$ & $2.30(1.24-4.26)$ \\
\hline PPI total ${ }^{\ddagger}$ & $141.5(15.06)$ & $.91(.68-1.20)$ & $.99(.71-1.38)$ & $1.13(.75-1.71)$ \\
\hline Total delinquency & $31.26(20.76)$ & $1.22(.84-1.78)$ & $.92(.59-1.43)$ & $.87(.53-1.42)$ \\
\hline
\end{tabular}

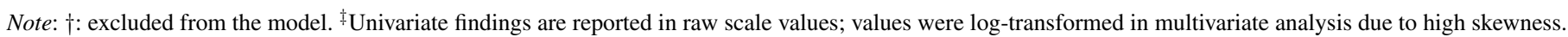
AOR: adjusted odds ratio, CI: confidence interval, Ref: reference group. Values in bold are statistically significant based on a $95 \%$ CI.

a $\mathrm{AIC}=434.78$, residual deviance $=390.78$.

b $\mathrm{AIC}=737.20$, residual deviance $=707.20$

c $\mathrm{AIC}=507.63$, residual deviance $=477.63$.

incarceration for youth ranged from 0 to 60 months, $($ mean $=7.5$, S.D. $=8.5)$. Approximately $37 \%(n=93)$ of youth reported having friends or siblings who used inhalants, and 38\% ( $n=106)$ reported having a problem with inhalants prior to incarceration. Table 1 provides a univariate summary of the other study measures.

\subsection{Summary of perceived harm and intentions of future inhalant use}

Approximately $38 \%$ of youth indicated that experimental use of inhalants was associated with a great risk of harm, $24 \%$ per- ceived a medium risk, 27\% perceived a slight risk, and $10 \%$ perceived no risk (see Table 1). A higher percentage of youth perceived regular inhalant use to be associated with great risk of harm (71\%). Approximately $18 \%$ believed regular inhalant use was associated with a medium risk, and only small percentages believed regular inhalant use was associated with slight (7.2\%) or no risk (4.7\%). A Spearman rank correlation showed the measures of perceived harm to have a moderately strong correlation $(\rho=.62, p<.0001)$.

The majority of adolescent inhalant users reported no intention of future inhalant use (74\%). Approximately $16 \%$ perceived a small likelihood, and $7 \%$ perceived an even chance. A small 
percentage reported an elevated likelihood of future use - that is, $3 \%$ perceived a good chance, and $1 \%$ was "sure to use." A Spearman rank correlation showed intention of future use to have a weak negative association with perceived risk of harm with experimental inhalant use $(\rho=-.12, p=.05)$, and a nonsignificant association with perceived risk of harm with regular inhalant use $(\rho=-.03, p=.60)$.

\subsection{Multivariate associations}

Multivariate ordinal logistic regression was used to identify factors associated with perceived risk of harm with using inhalants and intentions of future inhalant use among youth with lifetime inhalant use. Table 1 summarizes odds ratios for the three models that were specified. Contrary to the study hypothesis, only one variable was associated with perceived risk of harm. Specifically, youth who reported having friends or siblings who used inhalants were significantly more likely to report a lower risk of harm compared to youth without friends or siblings who used inhalants. This variable was significant for both risk of harm with experimental use of inhalants $(\mathrm{OR}=.55$, $95 \% \mathrm{CI}=.34-.89)$ and regular use of inhalants $(\mathrm{OR}=.47,95 \%$ $\mathrm{CI}=.47,95 \% \mathrm{CI}=.26-.82$ ).

Youth with friends or siblings who used inhalants were over three times more likely to report a higher likelihood of future inhalant use compared to youth without these network influences $(\mathrm{OR}=3.33,95 \% \mathrm{CI}=1.77-6.29)$. Being younger, reporting greater current psychiatric distress, evidencing more extensive substance abuse problem histories including prior problems with inhalants also increased likelihood of future inhalant use. Contrary to our hypothesis, there was no evidence that perceptions of harm were significantly associated with perceived likelihood of future inhalant use.

\section{Discussion}

Nearly $90 \%$ of adolescent inhalant users perceived regular inhalant use as a moderately-to-highly risky activity. Yet, more than one-quarter of youth reported some likelihood of future inhalant use. This figure may be significantly underestimated if youth perceived some incentive to minimize or misrepresent their future inhalant use intentions. Given that inhalant use is strongly influenced by proximal social contexts (Vaughn et al., 2007) and peer influences (Perron et al., in press), it is also possible that youth perceived little likelihood of future inhalant use within the study setting, but that contextual factors could operate to increase risk for inhalant use in the future. This is particularly relevant given that perceptions of harm of inhalant use were uncorrelated with intentions to use, but social networks exhibited a stable association.

Inhalant prevention and treatment efforts should focus on important and easily assessed correlates of future intentions to use inhalants including self-reports of a prior problem with inhalants and social networks including family and friends who use inhalants. Adolescent inhalant users who are younger, in greater psychiatric distress, and with more extensive substance use problems were also easily identified groups at risk for future inhalant use in this juvenile justice population (Howard et al., 2008; Wu and Howard, 2007). In light of the substantial prevalence and seriously adverse consequences of inhalant use within this population (Sakai et al., 2004; Storr et al., 2007; Williams and Storck, 2007), it is critical that juvenile justice programs implement effective methods for identifying and intervening with youth who are at risk for commencing, maintaining, and intensifying inhalant use following discharge from treatment.

Strengths of this investigation included the large sample of inhalant users, use of face-to-face structured interviews, high study participation rate, examination of an important and understudied clinical population at high risk for inhalantrelated problems, and focus on an under researched issue. Limitations include the self-report nature of the assessments and possibly limited generalizability of study findings given that the sample was comprised of youth who are incarcerated.

\section{Acknowledgements}

This study was supported by grants DA021405 (Natural History, Comorbid Mental Disorders, and Consequences of Inhalant Abuse, M.O. Howard, PI), and DA15929 (Neuropsychiatric Impairment in Adolescent Inhalant Abusers, M.O. Howard, PI) from the National Institute on Drug Abuse.

Role of funding source: NIDA had no further role in study design; in the collection, analysis and interpretation of data; in the writing of the report; or in the decision to submit the paper for publication.

Contributors: The second author of this study designed the survey and supervised the collection of the data and assisted with statistical analyses. Both authors jointly conceptualized this study and prepared the manuscript.

Conflict of interest: Neither author has any financial interests or relationships relevant to the subject of this manuscript.

\section{References}

Balster, R.L., 1997. Inhalant abuse: a forgotten drug abuse problem. In: College on Problems of Drug Dependence Presidential Address. Conference Proceedings, NIDA Research Monograph, 174, pp. 3-8.

Beauvais, F., 1992. Attitudes about drugs and the drug use of Indian youth. Am. Indian Alsk Native Ment. Health Res. 5, 38-42.

Crano, W.D., Gilbert, C., Alvaro, E.M., Siegel, J.T. Enhancing predictor of inhalant abuse risk in samples of early adolescents: a secondary analysis. Addict. Behav., in press.

Derogatis, L.R., 1993. BSI bibliography. Minneapolis: National Computer Systems.

Elliott, D.S., Huizinga, D., Menard, S., 1989. Multiple Problem Youth: Delinquency, Substance Use and Mental Health Problems. Springer-Verlag, New York.

Grisso, T., Barnum, R., 2000. Massachusetts Youth Screening Instrument Second Version: User's Manual and technical Reports. University of Massachusetts Medical School, Boston, MA.

Howard, M.O., Jenson, J.M., 1999. Inhalant use among antisocial youth: prevalence and correlates. Addict. Behav. 24, 59-74.

Howard, M.O., Walker, R.D., Walker, P.S., Cottler, L.B., Compton, W.M., 1999 Inhalant use among urban American Indian youth. Addiction 94, 83-95.

Howard, M.O., Balster, R.L., Cottler, L.B., Wu, L.T., Vaughn, M.G., 2008. Inhalant use among incarcerated adolescents in the United States: preva- 
lence, characteristics, and correlates of use. Drug Alcohol Depend. 93, 197-209.

Johnson, L.D., O’Malley, P.M., Bachman, J.G., Schulenberg, J.E., 2007. Monitoring the Future national survey results on drug use, 1975-2006. Volume I: Secondary School Students (NIH Publication no. 07-6205). Bethesda, MD: National Institute on Drug Abuse.

Kissin, D.M., Zapata, L., Yorick, R., Vinogradova, E.N., Volkova, G.V., Cherkassova, E., Lynch, A., Leigh, J., Jamieson, D.J., Marchbanks, P.A., Hillis, S., 2007. HIV seroprevalence in street youth, St. Petersburg, Russia. AIDS 21, 2333-2340.

Lilienfeld, S.O., Andrews, B.P., 1996. Development and preliminary validation of a self-report measure of psychopathic personality traits in noncriminal populations. J. Pers. Assess. 66, 488-524.

Medina-Mora, M.E., Real, T., 2008. Epidemiology of inhalant use. Curr. Opin. Psychiatry 21, 247-251.

Perron, B.E., Vaughn, M.G., Howard, M.O. Reasons for using inhalants: Evidence for discrete classes in a sample of incarcerated adolescents. J. Subst. Abuse Treat., in press.

Sakai, J.T., Hall, S.K., Mikulich-Gilbertson, S.K., Crowley, T.J., 2004. Inhalant use, abuse, and dependence among adolescent patients: com- monly comorbid problems. J. Am. Acad. Child Adolesc. Psychiatr. 43, $1-12$.

Shimane, T., Wada, K., 2007. Substance use among night high school students in Japan. Nihon Arukoru Yakubutsu Igakkai Zasshi 42, $152-164$.

Storr, C.L., Accornero, V.H., Crum, R.M., 2007. Profiles of disruptive behavior: association with recent drug consumption among adolescents. Addict Behav. $32,248-264$

Vaughn, M.G., Perron, B.E., Howard, M.O., 2007. Variations in social contexts and their effect on adolescent inhalant use: a latent profile investigation. Drug Alcohol Depend. 91, 129-133.

Williams, J.F., Storck, M., Committee on Substance Abuse and Committee on Native American Child Health, 2007. Inhalant abuse. Am. Acad. Pediatr. 119, 1009-1017.

Wu, L.T., Howard, M.O., 2007. Psychiatric disorders among inhalant users: findings from the National Epidemiologic Survey on Alcohol and Related Conditions. Drug Alcohol Depend. 88, 146-155

Wu, L.T., Pilowsky, D.J., Schlenger, W.E., 2004. Inhalant abuse and dependence among adolescents in the United States. J. Am. Acad. Child Adolesc. Psych. 43, 1206-1214. 